\title{
LA PENÍNSULA IBÉRICA EN LA PLANIFICACIÓN MILITAR ALIADA EN 1942-1943
}

\author{
Antonio Marquina ${ }^{1}$ \\ Director de UNISCI
}

\section{Title in English: "The Iberian Peninsula in the Allied Military Planning"}

\author{
Copyright @ C UNISCI, 2014.
}

Las opiniones expresadas en estos artículos son propias de sus autores, y no reflejan necesariamente la opinión de UNISCI. The views expressed in these articles are those of the authors, and do not necessarily reflect the views of UNISCI.

\section{Introducción}

Una vez que los aliados tomaron la decisión de llevar a efecto la operación Torch, las implicaciones de la neutralidad o beligerancia de la Península Ibérica pasaron a primer plano. Ya hemos expuesto someramente en uno de nuestros trabajos las evaluaciones y preparativos para este último supuesto, el así denominado plan Backbone. Procedamos a clarificar este panorama un poco más.

En agosto de 1942 el Comité Estratégico Conjunto norteamericano consideró que eran impracticables operaciones de envergadura contra el noroeste de Europa antes de 1944, dadas las constricciones que imponían las disponibilidades logísticas. Al mismo tiempo se analizó con bastante extensión si las operaciones ofensivas en la Península Ibérica eran aconsejables, factibles y aceptables. En caso positivo tendrían que formular una directiva sobre ellas.

El estudio hizo un repaso de los factores políticos, económicos y psicológicos de España y Portugal; la situación de las fuerzas armadas, disposición, eficiencia, moral, equipo, entrenamiento y teorías de combate; las posibilidades de refuerzo por los países del Eje; y un análisis de las características de la Península Ibérica: geografía, hidrografía, clima, situaciones relativas y distancias, líneas de transporte y abastecimiento, fortificaciones, capacidades de los puertos e. instalaciones aéreas.

\footnotetext{
${ }^{1}$ Antonio Marquina Barrio es Catedrático de Seguridad y Cooperación en las Relaciones Internacionales de la Universidad Complutense de Madrid, Director del Departamento de DIP y Relaciones Internacionales de la UCM, Director de UNISCI y Presidente del Foro Hispano-Argelino. Sus principales líneas de investigación son la seguridad en Europa, el Mediterráneo y Asia-Pacífico, y el control de armamentos.

Dirección: Departamento de Estudios Internacionales, Facultad de Ciencias Políticas y Sociología, UCM, Campus de Somosaguas, 28223 Madrid, España.

E-mail: marioant@cps.ucm.es.
} 
Estudiados todos estos asuntos, la solución del problema era claramente negativa. Una operación aliada limitada únicamente a la Península Ibérica sería inaceptable, pues las ganancias no tendrían correspondencia con el esfuerzo realizado. La operación se había de efectuar si significaba la apertura de un nuevo frente en una campaña decisiva contra el Eje, ejecutada principalmente por las fuerzas aéreas y terrestres de Estados Unidos con el apoyo naval británico y norteamericano. Una operación de poca envergadura se evaluó como desaconsejable y peligrosa. El objetivo último habría de ser forzar los Pirineos y, con ello, obligar al Eje a reorganizar su defensa en Francia, aliviando la presión sobre Rusia y Oriente Medio, apoyando, asimismo, indirectamente, la defensa de las islas británicas. Pero el Comité Estratégico Conjunto concluyó que la conquista de la Península Ibérica no afectaría decisivamente a ninguno de estos objetivos esenciales, por lo que su contribución a la derrota del Eje sería limitada.

Esta operación tampoco sería muy viable, ya que los países del Eje podían desplegar en este teatro una fuerza superior a las 20 divisiones iniciales previstas en los planes americanos, dada su mayor cercanía, y, por otra parte, la dificultad de hacer llegar refuerzos sería considerable por las limitaciones de las instalaciones portuarias, el estado y trazado de las carreteras y el ferrocarril, y sobre todo porque había que cruzar los Pirineos, que podrían bloquearse fácilmente por el Eje. La Península no constituiría un área defensiva tan buena para los aliados como el norte de África. Además, era casi seguro que España y Portugal resistirían una invasión colocándose en la órbita del Eje. En resumen, las ganancias serían pequeñas y obligarían a una operación larga en un teatro que no era decisivo. Por lo tanto no habrían de llevarse a efecto operaciones ofensivas en la Península.

Una vez realizada la operación $\operatorname{Torch}^{2}$, se volvió a reconsiderar la situación y la planificación para las operaciones subsiguientes en el Mediterráneo. La idea básica consistía en la apertura de las líneas marítimas de comunicación en el Mediterráneo explotando el éxito obtenido en Torch y en las batallas del desierto libio. Los Estados Mayores británico y norteamericano estudiaron las líneas de acción posibles del Eje en respuesta a la nueva situación creada por los desembarco s aliados. A su juicio los posibles movimientos eran los siguientes:

1. La defensa directa de sus posiciones en el Mediterráneo.

2. Un ataque a través de Turquía y/o Persia en las instalaciones petrolíferas de Oriente Medio y las líneas de comunicación.

3. Un ataque a través de España para conseguir obtener la zona norte del estrecho de Gibraltar.

Esta última posibilidad se consideraba ya a finales de noviembre de 1942 «extremadamente dudosa», concluyéndose que lo más probable sería que el Eje se contentase con defender sus posiciones en el Mediterráneo, si bien no había de perder de vista los peligros latentes en los flancos de este teatro.

La estrategia aliada en este momento consistía en debilitar al máximo el poder militar alemán antes de empeñarse en una operación a gran escala de reentrada en el continente europeo. Por ello, para agravar el compromiso del Eje en el Mediterráneo, se consideraron tres posibilidades:

1. Invasión de los Balcanes.

2. Invasión de España.

\footnotetext{
${ }^{2}$ Se trata, como es sabido, de la operación de desembarco en el norte de África por las tropas aliadas iniciada en noviembre de 1942 .
} 


\section{Invasión de Italia.}

La invasión de España se desechó de inmediato, por las razones anteriormente apuntadas, considerándose la invasión de Italia como una gran oportunidad para complicar y debilitar el poderío militar alemán.

Sin embargo, se siguió todavía considerando la posibilidad de movimientos alemanes en España que había que prevenir. Describiremos las dos operaciones barajadas y hechas llegar al general Wedemeyer en enero de 1943. Estas operaciones en la Península tendrían como finalidad asegurar las líneas de comunicación aliadas a través del estrecho de Gibraltar y el establecimiento de bases aéreas desde donde desplegar todo tipo de operaciones contra el Eje.

\section{El plan de 36 divisiones}

Este plan se dividió en cinco fases, suponiendo que las tropas necesarias para llevarlo a efecto estuviesen previstas y asignadas, se iniciase con anterioridad la ocupación del Marruecos español cuando la colaboración española con el Eje pareciese inminente, y que la invasión del Eje no ocurriese antes de que el plan aliado estuviese en desarrollo. Se contaba también con mantener el control del litoral del norte de África, la continuación de operaciones aéreas en el Mediterráneo central y con que Rusia contendría el grueso de las fuerzas alemanas.

La primera fase establecía desembarcos principales en las zonas de Lisboa, Huelva, Cádiz y Almería, y desembarcos menores en Málaga y Campo de Gibraltar, hasta asegurar un frente que abarcase desde el norte de Coimbra hasta Almería, pasando por Badajoz, Zafra y Córdoba. La segunda consistía en el avance desde aquella línea hasta otra línea ondulada que abarcaba desde el norte de Oporto hasta Gandía, pasando por Ciudad Rodrigo, Navalmoral de la Mata y Ciudad Real; se incluían nuevos desembarcos en Cartagena, Alicante y Oporto. La tercera fase suponía un avance desde esta línea hasta la fijación de una tercera línea que cubría gran parte de Castilla la Vieja, descendiendo por la provincia de Cuenca hasta Valencia. La cuarta fijaba la línea en la cuenca del Ebro. Y la última fase hacía llegar las fuerzas aliadas a los Pirineos a través de Vitoria-San Sebastián, Huesca, Jaca y otra línea de penetración al este a través de Lérida y Barcelona.

Este plan de gran escala requería 36 divisiones, de las cuales 8 deberían estar preparadas para el asalto (6 anfibias y 2 acorazadas), 611 barcos de transporte, de los cuales 60 habrían de utilizarse en el período inicial, 778 barcos de carga, utilizándose también 60 en la primera fase, y cerca de 150 lanchas de desembarco.

De todas estas fuerzas requeridas para la operación, sólo las lanchas de desembarco estarían disponibles durante el año de 1943, concluyéndose que este tipo de operación no podría llevarse a efecto hasta después de 1943. Se evaluaron también las ventajas y los inconvenientes. Las ventajas eran claras:

1. Las líneas de comunicación aliadas a través del estrecho de Gibraltar estarían aseguradas contra las amenazas del Eje desde la Península.

2. Se asegurarían bases navales y aéreas para operaciones ulteriores contra el Eje.

3. Se reforzaría el bloqueo contra el Eje.

4. Se conseguiría una plataforma para posteriores operaciones combinadas que se harían coincidir con una invasión de Europa a través del canal de la Mancha.

Pero los inconvenientes eran más numerosos y de mayor entidad: 
1. Elevado coste de los desembarcos en una costa hostil.

2. Pérdida de embarcaciones.

3. Sería la más importante operación aliada durante 1943, comprometiendo grandes fuerzas, que serían muy difíciles de mantener logísticamente.

4. Implicaría un gran esfuerzo en el transporte para mantener las líneas de comunicación interiores.

5. Conllevaría una gran vulnerabilidad de las líneas de comunicación peninsulares a los ataques aéreos.

6. Dada la pobreza del país, ello constituiría un compromiso económico y militar para Estados Unidos.

7. Dadas las capacidades portuarias, el transporte de todas las unidades a la Península llevaría veinte meses.

8. La envergadura de esta operación pospondría indefinidamente la invasión a través del Canal de la Mancha.

Todos estos inconvenientes se reiterarán, como veremos, y explican, frente a otras opiniones menos avisadas, el desinterés aliado por el teatro español y el interés por la "neutralidad" española.

\section{Plan para control de una cabeza de puente en el sur de España}

Este plan alternativo tenía también como finalidad asegurar el estrecho de Gibraltar en el caso de que España empezase a colaborar activamente con el Eje y Alemania estuviera preparándose para iniciar un movimiento de fuerza en la Península Ibérica. Los aliados tomarían la iniciativa de la invasión para asegurar los puertos necesarios para el desembarco rápido de las fuerzas. Desde Gibraltar se desplegarían previamente fuerzas ligeras en la zona de Tarifa.

Tres fases comprendía este plan. En la primera, se llevarían a efecto bombardeos desde las bases en el Reino Unido y el norte de África para cortar las líneas de comunicaciones en España, impidiendo los movimientos de las fuerzas del Eje. En la segunda, tendrían lugar los desembarcos de fuerzas anfibias en las zonas de Huelva, Cádiz, Málaga y Almería, avanzando rápidamente para asegurar una línea defensiva a lo largo de las montañas de Sierra Morena. Finalmente, se fijaría una línea de defensa y se procedería a un rápido incremento de fuerzas.

Este plan había sido propuesto en sus líneas esenciales por algunos miembros de la junta militar aliadófila española formada en 1940 y apoyada económicamente por Inglaterra. Para esta operación serían necesarias 15 divisiones:

1. Cuatro divisiones anfibias (60.000 hombres).

2. Dos divisiones acorazadas (30.000 hombres).

3. Nueve divisiones de infantería (135.000 hombres).

4. Tropas de apoyo (200.000 hombres).

A esta fuerza había que añadir cuatro grupos de caza, dos grupos de bombarderos ligeros y la utilización en un primer momento de 206 barcos, entre barcos de transporte, de carga y de desembarco, a los que seguirían 231 barcos de transporte y 268 de carga. Esta operación utilizaría por completo las tropas disponibles de los Estados Unidos.

La evaluación de la operación en su conjunto fue muy significativa. Con la conquista del sur de la Península se conseguía la protección de Gibraltar ante posibles ataques por tierra y, en 
no menor medida, la reducción de la amenaza aérea a los convoyes que transitasen por el estrecho. A la vez, Alemania se vería obligada a ocupar la mayor parte de España con todos los compromisos subsiguientes militares y económicos, el despliegue de sus fuerzas aéreas y terrestres a expensas de otros frentes, con unas líneas de comunicación deficientes y bastante vulnerables a la aviación, de modo peculiar en los pasos pirenaicos. Además, se contaba con el apoyo de los comunistas (principales contactos de la OSS) para la realización de acciones subversivas.

Frente a estas ventajas indudables, los inconvenientes eran notables: Alemania podría ocupar las islas Baleares, adquiriendo con ello bases aéreas en el Mediterráneo occidental; la línea defensiva en Sierra Morena sería difícil de mantener, al no constituir una barrera natural de importancia, contando con las tácticas modernas ofensivas alemanas. El colapso de esta línea sería una cuestión de tiempo, a menos que se reforzasen significativamente las fuerzas desembarcadas y se intentara la conquista total de España. Las fuerzas del Eje podrían reforzar mucho más rápidamente el teatro peninsular antes que los aliados. Con todo, la realidad era mucho más compleja que un cálculo de posibilidades bastante voluntarista. La realización de esta operación implicaría el compromiso casi completo en hombres y barcos disponibles durante cuatro a seis meses en perjuicio de las operaciones ofensivas en otros teatros, y pospondría indefinidamente los preparativos para el cruce del Canal de la Mancha y el ataque al teatro decisivo de la guerra. La conquista del teatro peninsular no implicaría de suyo una entrada decisiva en el continente europeo, los Pirineos lo impedirían.

A estos borradores se añadió una evaluación de la posible amenaza española a las operaciones ofensivas aliadas en el Mediterráneo. Los servicios de inteligencia no pudieron encontrar signos evidentes de que Alemania estuviera concentrando tropas en el sur de Francia para invadir a España o que España intentase colaborar con el Eje, aunque existían múltiples indicaciones de que Alemania estaba haciendo grandes esfuerzos para hacer creer a los aliados que su invasión de España era inminente, a la vez que fomentaba entre los españoles la desconfianza en los aliados.

Las conclusiones de este informe fueron bastante tranquilizadoras. España no colaboraría con el Eje en una operación militar contra los aliados, ya que deseaba mantenerse fuera de la guerra por razones económicas y de integridad territorial, dada la situación de las islas Canarias. Alemania, por su parte, dados sus compromisos en Rusia, sus refuerzos a Italia, los Balcanes y el norte de África, no podría entrar en España sin una activa colaboración española. Sin embargo, en el supuesto de que Alemania, haciendo las necesarias reducciones en diversos frentes, fuese capaz de reunir las suficientes fuerzas para invadir España, sería factible que alcanzasen el sur peninsular antes que las fuerzas aliadas pudieran desembarcar, a menos que estuviesen preparadas con antelación, lo cual no era factible en ese momento por falta de barcos. Esto preocupaba y esta era la eventualidad que había que prevenir, aunque se estimaba, como señalamos, que todas las indicaciones acerca de una inminente invasión alemana eran puros rumores hechos circular por los agentes alemanes para inmovilizar gran número de fuerzas aliadas ante esta supuesta amenaza.

\section{Operaciones de apovo a Portugal}

La operación Torch va a traer también complicaciones a Portugal, que van a tener un inmediato reflejo en España. En Washington, los Estados Mayores británico y norteamericano consideraban necesaria la utilización del archipiélago de las Azores para la lucha antisubmarina, reabastecimiento de barcos y lugar de parada de los aviones en tránsito a Europa y Asia. 
El presidente Roosevelt y el primer ministro Churchill en su reunión de Casablanca discutieron el uso de las Azores. Churchill, aconsejado por Eden, se mostró cauteloso ante una ocupación del archipiélago sin el consentimiento de Portugal. Nuevamente vuelven a encontrarse ambos estadistas en Washington en el mes de mayo para la Conferencia Tridente. Roosevelt instó a Churchill a conseguir los objetivos recurriendo a los hechos consumados y Churchill aceptó la sugerencia. El Estado Mayor norteamericano preparó los planes de desembarco.

El 24 de mayo, el Gabinete de Guerra británico concluyó que era necesario consultar con Salazar previamente y así se lo telegrafiaron a Churchill, pero Roosevelt accedió a regañadientes, dando un plazo al primer ministro para que reexaminasen el problema. Finalmente los británicos decidieron una acción diplomática previa ante Salazar, una semana antes de realizar la operación. Salazar recibió la propuesta del embajador británico en Lisboa, Ronald Campbell, el 18 de junio y dio su aprobación de principio el 23 de junio. El presidente Roosevelt, en el interim, mandó preparar un estudio teniendo en cuenta los siguientes presupuestos:

1. Portugal no sólo da su conformidad a la ocupación pacífica de las Azores, al ser solicitado su apoyo por el gobierno británico, sino que expresa además su intención de declarar la guerra contra el Eje.

2. Las Naciones Unidas han de suministrar algún tipo de apoyo a Portugal. En su opinión bastaría con 40.000 hombres.

3. Este apoyo no debía interferir o perjudicar los planes Overlord o Pointblank.

La Junta de Jefes de Estado Mayor ordenó los estudios correspondientes, que llevaron por título Operations to assist Portugal. El Estado Mayor Conjunto de Planificación elevó, el 26 de junio de 1943, a la Junta de Jefes de Estado Mayor un informe secreto que tuvo una distribución restringida, once copias en total. Este estudio incluía un memorándum para el presidente, un borrador de carta del presidente Roosevelt al primer ministro británico y un plan sumario para llevar a efecto una campaña de importancia en la Península Ibérica, este último para información de la Junta de Jefes de Estado Mayor.

Para el Estado Mayor Conjunto de Planificación, el alineamiento de Portugal con los aliados y la entrada de tropas en Portugal proporcionaría un apoyo político y psicológico a la causa de las Naciones Unidas. Sin embargo, era deseable obtener el permiso de utilización de las islas Azores únicamente.

Las razones eran bastante claras. La utilización del territorio continental portugués ciertamente proporcionaría bases mejor colocadas para la realización de ofensivas aéreas contra los puertos del sur del golfo de Vizcaya, mejor protección para las líneas marítimas del Mediterráneo, facilidades aéreas para la ruta Reino Unido-Africa y mejor efectividad en el bloqueo y lucha antisubmarina. Pero los inconvenientes eran de gran envergadura. Portugal solicitaría a los aliados la aportación de fuerzas defensivas en el territorio continental, que probablemente habrían de incrementarse, y la reacción aérea del Eje en contra tendría más entidad. Cualquier incremento de fuerzas afectaría las operaciones proyectadas como Overlord, Pointblanc y Priceless y sobre todo se correría el riesgo de iniciar una campaña en la Península Ibérica, una zona no decisiva, donde la logística era muy difícil y en la que la barrera de los Pirineos impediría la explotación del éxito, con la posposición de Overlord y el abandono de Priceless.

En el caso de que Alemania tomase la decisión de invadir España como consecuencia de la declaración de guerra de Portugal, los preparativos al efecto en el sur de Francia se harían patentes a las dos semanas, estimándose que el total de tropas concentradas se había de elevar 
a 20 divisiones, 525 aviones de combate y 525 aviones de transporte. Lo más probable era que España resistiese la invasión y contuviese a los alemanes al menos una semana en los Pirineos. La línea constituida por el valle del Ebro no podría ser alcanzada antes de las tres semanas. Eran probables desembarcos en la costa este, norte y en Baleares. Sobre estos presupuestos, una vez desembarcados 40.000 hombres en Portugal para su apoyo y defensa, las operaciones aliadas se estructuraban en cuatro fases.

La primera implicaba el bombardeo de los pasos de los Pirineos desde el norte de África y el Reino Unido, el transporte aéreo de una división desde el norte de África a Barcelona y Zaragoza en apoyo a las fuerzas españolas en el nordeste del río Ebro, así como el avance de las fuerzas destacadas en Portugal hacia Bilbao para asegurar el flanco de la línea del Ebro. Además, el transporte aéreo inmediato de un regimiento a Baleares para el apoyo de su defensa y el estacionamiento de unidades aéreas en España y Portugal. Todo esto había de llevarse a efecto en dos semanas, o tres como máximo.

La segunda fase se iniciaba con el transporte de una división estacionada en el norte de África a Baleares, y de otras tropas a los puertos de la costa sur y este de España, utilizando todos los medios de desembarco disponibles en el Mediterráneo. Se continuaría el refuerzo de las unidades españolas que resistiesen en el valle del Ebro, especialmente en el nordeste, el incremento de las fuerzas aéreas en la Península e islas Baleares y se iniciarían los desembarcos de tropas provenientes de los Estados Unidos en los puertos atlánticos de la Península. En la tercera fase se completarían los refuerzos hasta un mínimo de 30 divisiones y 2.000 aviones. Las fuerzas del Eje retrocederían hasta estar fuera de la Península. La cuarta fase culminaba con el control de los pasos pirenaicos y la preparación para iniciar operaciones en Francia.

Los planificadores volvían a reiterar que estas operaciones eran muy costosas, tratándose de una zona no decisiva para la derrota del Eje. Por ello, el Estado Mayor Conjunto de Planificación indicó que había que desaconsejar a Portugal la declaración de guerra al Eje, haciéndole ver que la aportación de fuerzas defensivas por los aliados no favorecería la causa de las Naciones Unidas, opinión que compartía el primer ministro Churchill.

El Comité Conjunto de Planes de Guerra estudió este informe y preparó algunas recomendaciones de cambios. Su opinión no varió con respecto a las implicaciones que podría traer consigo una declaración de guerra al Eje por Portugal. La Junta de Jefes de Estado Mayor discutió el informe del Estado Mayor Conjunto de Planificación. El general Marshall opinó que o bien se había de evitar entrar en Portugal continental o bien había que hacerlo con una fuerza apropiada. Una fuerza de 40.000 hombres era insuficiente para una defensa adecuada que exigiría 80.000 hombres y, por otra parte, debían aceptar la posibilidad de interferencias con otras operaciones ya proyectadas si se quería asegurar la capacidad de maniobra en la prestación del apoyo necesario. El almirante King subrayó los perjuicios que se producirían en la preparación de Overlord con el envío de fuerzas a Portugal, aun admitiendo los beneficios que reportaría contar con instalaciones en Portugal. Esta última opinión fue compartida por el general Marshall y el general Arnold. El general Arnold resaltó la posibilidad de un ataque alemán a través de España y los almirantes Leahy y Willson mostraron su seguridad en un ataque alemán, por lo que habría que contar con el envío de fuerzas para la defensa de Portugal. La Junta de Jefes de Estado Mayor ordenó una reelaboración del memorandum para el presidente, un estudio de los resultados beneficiosos que la ocupación de Portugal había de reportar y una clarificación de la actitud de Churchill con respecto a la posición que había de adoptar Portugal, declarar o no declarar la guerra.

Finalmente se envió el memorandum corregido al presidente Roosevelt. En él se resumieron estas observaciones subrayándose desde el principio que el envío de una fuerza de las 
Naciones Unidas, aunque fuese pequeña, a Portugal podría precipitar una invasión de España por el Eje, aunque se consideraba improbable. En este supuesto la campaña en la Península Ibérica sería de tono mayor, implicando la utilización de 30 divisiones de las Naciones Unidas y 2.700 aviones. Esta pospondría indefinidamente Overlord y cortaría drásticamente otras operaciones menores en el Mediterráneo. Luego se incidía en la asistencia y adecuada defensa de Portugal que implicaría el empleo del doble número de fuerzas consideradas por el presidente, resaltando las dificultades que entrañaba el movimiento de una fuerza de este calibre y las implicaciones inevitables en Overlord, Pointblank y Priceless. El coste estimado en la preparación de Overlord era de 2 a 4 divisiones.

Este coste había que aceptarlo. En el mensaje propuesto para el primer ministro se volvían a reiterar estas ideas y la necesidad de aceptar ciertos retrasos en las operaciones acordadas en Tridente. Finalmente, acabó imponiéndose el criterio de la diplomacia británica de no forzar la situación ante la tozudez de Salazar, llegándose sólo a un acuerdo entre Portugal y el Reino Unido en función de la antigua alianza luso-británica. Los norteamericanos hubieron de conformarse, tras un durísimo forcejeo y largas dilaciones, con el permiso, en febrero de 1944, para la construcción de un aeropuerto que pudiera interesar a la aviación civil en un futuro no lejano.

Nos hemos detenido en estas negociaciones, independientemente de su incidencia en los planes militares aliados acerca de la Península, para dar alguna dimensión a la compleja realidad de la planificación militar y resaltar los objetivos primarios que los aliados mantenían en este momento, así como los matices diferentes de Inglaterra y Estados Unidos. A partir de estas negociaciones y, más en concreto, a partir de agosto de 1943, ya no queda constancia documental de planes de invasión en la Península. Antes de la invasión de Normandía, la Junta de Jefes de Estado Mayor norteamericana consideró deseable la entrada de España y Portugal en guerra contra el Eje, pero ya el tratamiento era distinto, conseguir ventajas sin grandes requerimientos de apoyo.

Esta amplia exposición creo que desmonta de una vez las aberraciones de libros como el del diplomático José María Doussinague, nada menos que director general de Política Exterior en el ministerio de Asuntos Exteriores, titulado España tenía razón, del cual han bebido con entusiasmo y acríticamente conocidos historiadores del franquismo. Terminaremos con una cita, entre otras, de este libro:

En efecto, llegaron a Madrid noticias de un informe secretísimo, conocido de muy pocas personas en Washington. En él se afirmaba la invulnerabilidad de las defensas alemanas que rodeaban el continente europeo, no hallándose más solución para establecer el segundo frente continental que volver a la idea del plan Imoff y atacar a España y Portugal con la ayuda de elementos revolucionarios que, en efecto, se movilizaron nuevamente dentro de la Península. Esta vez el plan se tomó muy en serio, y el corte de suministros petrolíferos debía considerarse como una medida previa a su puesta en ejecución.

Las informaciones que fueron llegando sucesivamente eran precisas: hubo comunicaciones... Stalin exigió que se cumpliera lo prometido en Teherán y se atacara de frente el cinturón de fortificaciones alemanas de Francia, con la esperanza de que los ingleses y americanos se estrellaran contra la resistencia alemana, dando lugar a que las tropas rusas pudieran ocupar toda Alemania... En Madrid se tuvo conocimiento detallado de todo lo que se planeaba y se conoció esa negativa de Stalin ${ }^{3}$.

\footnotetext{
${ }^{3}$ Una visión más completa de estos acontecimientos se puede encontrar en Marquina Antonio (1986): España en la Política de Seguridad Occidental 1939-1986, Madrid. Ed. Ejército.
} 